\title{
Importancia de un diagnóstico oportuno del carcinoma oral de células escamosas. Reporte de caso clínico y revisión de la literatura
}

\section{Importance of a timely diagnosis of oral squamous cell carcinoma. A case report and review of the literature}

\section{Resumen}

Se resalta la relevancia de un diagnóstico oportuno en el cáncer de cavidad oral, así como una breve síntesis de la literatura. Se reporta el caso de una paciente que acude a la clínica de Cirugía Oral y Maxilofacial de la UNAM con antecedentes de habérsele realizado varias biopsias en las cuales no se encontraron resultados concluyentes. Por lo cual se decide realizar la toma de biopsia incisional para poder definir la patología. El artículo tiene como objetivo hacer énfasis en la importancia de un diagnóstico correcto y a tiempo, para que el paciente pueda recibir el mejor tratamiento. En conclusión, el papel que desempeña el especialista en una detección temprana y acertada y el compromiso en conjunto del paciente, puede disminuir considerablemente la morbimortalidad.

Palabras clave: Carcinoma; Mandíbula; Biopsia (fuente: DeCS BIREME).

\section{Caso Clínico}

Andrés Gabriel Larreta Gutiérrez ${ }^{1, a}$, Fabiola Salgado Chavarría $^{1, b}$, Mauricio Antonio Velasco Tizcareño ${ }^{1, c}$

${ }^{1}$ Universidad Nacional Autónoma de México, Facultad de Odontología, División de Estudios de Postgrado e Investigación, Especialidad de Cirugía Oral y Maxilofacial, Ciudad de México, México.

a Cirujano Dentista.

b Doctora en Ciencias Médicas, Odontológicas y de la Salud.

${ }^{\mathrm{c}}$ Maestro en Educación.

\section{Correspondencia:}

Andrés Gabriel Larreta Gutiérrez: od_andreslarreta@ hotmail.com

Universidad Nacional Autónoma de México. Circuito de los Institutos s/n Ciudad Universitaria, Col. Copilco, Alcaldía, Coyoacán, 04510, Ciudad de México, México. ORCID: 0000-0002-5657-9635

\section{Coautores:}

Fabiola Salgado Chavarría: inv.cirugia-depei@fo.odonto. unam.mx

ORCID: 0000-0002-8507-8346

Mauricio Antonio Velasco Tizcareño: ens.cirugia-depei@ fo.odonto.unam.mx

ORCID: 0000-0003-2795-1386

\section{Editora:}

Marieta Petkova-Gueorguieva

Universidad Nacional Mayor de San Marcos, Perú.

Conflicto de intereses: los autores declaran no tener conflictos de interés.

Fuente de financiamiento: autofinanciado.

\section{Recibido: $11 / 05 / 20$}

Aceptado: 19/09/20

Publicado: $16 / 11 / 20$

\begin{abstract}
The relevance of a timely diagnosis in oral cavity cancer is pointed out, as well as a brief synthesis of the literature. The case of a female patient who attends the UNAM Oral and Maxillofacial Surgery Clinic with a history of having undergone several biopsies in which no conclusive results were found, is reported. Therefore, it was decided to perform an incisional biopsy in order to define the pathology. The article aims to emphasize the importance of a correct and timely diagnosis, so that the patient can receive the best treatment. In conclusion, the role played by the specialist in an early and accurate detection and the overall commitment of the patient can considerably reduce morbidity and mortality.
\end{abstract}

Keywords: Carcinoma; Mandible; Biopsy (source: MeSH NLM).

(C) Los autores. Este artículo es publicado por la revista Odontología Sanmarquina de la Facultad de Odontología, Universidad Nacional Mayor de San Marcos. Este es un artículo de acceso abierto, distribuido bajo los términos de la licencia Creative Commons Atribucion - No Comercia_Compartir Igual 4.0 Internacional. (http://creativecommons.org/licenses/by-nc-sa/4.0/) que permite el uso no comercial, distribución y reproducción en cualquier medio, siempre que la obra original sea debidamente citada. 


\section{Introducción}

El cáncer en cabeza y cuello engloba distintos tipos y estructuras anatómicas como la cavidad oral, orofaringe, laringe y nasofaringe ${ }^{1}$. Descrito por primera vez por el Dr. Dargent en 1945 quien realizó estudios de 59 pacientes ${ }^{2}$. El cáncer en la cavidad oral afecta principalmente: labios, lengua, mejillas, piso de boca, tejidos blandos y duros del paladar, y encías ${ }^{3}$. La detección en las etapas tardías del carcinoma oral de células escamosas (COCE) provee un pronóstico desfavorable para el paciente, ya que es un tipo de cáncer con una tasa de supervivencia ajustada a los cinco años que oscilan entre el 40\%-60\% del total de pacientes detectados ${ }^{4}$. La primera línea de detección del COCE está dada por una revisión rutinaria que incluye revisión intraoral, cuello y faringe que deben ser examinados minuciosamente en conjunto con estudios imagenológicos 5 .

Se considera como un factor de riesgo el consumo constante y excesivo de alcohol y tabaco ${ }^{6}$. El cáncer de cabeza y cuello es el sexto cáncer más común en todo el mundo y cerca del $40 \%$ de estos casos se diagnostican como cáncer oral (CO). Varios estudios han detectado que la población más afectada oscila entre la $4^{\text {ta }}$ y $6^{\text {ta }}$ década de vida, también se ha observado que es el cáncer más común en cavidad oral, con predominio en el sexo masculino ${ }^{7}$. El pronóstico del CO va a depender de su localización y etapas ${ }^{8,9}$. La Organización Mundial de la Salud (OMS) ha determinado que la detección temprana el cáncer oral es su principal objetivo ${ }^{9,10}$. El artículo tiene como objetivo reportar un caso de carcinoma oral de células escamosas de una paciente que acudió al Departamento de Cirugía Oral y Maxilofacial de la UNAM (Universidad Nacional Autónoma de México),

\section{Reporte del caso}

Se presenta paciente femenino de 61 ańos, originaria de Culiacán, Sinaloa, ubicada al Noroeste de México, de ocupación en el hogar, con una hija y con antecedentes personales heredofamiliares de cáncer negados, y refiere etilismo y tabaquismo ocasionalmente desde hace 20 años (Tabla 1), acude a la Clínica de Cirugía Oral y Maxilofacial de la Universidad Nacional Autónoma de México (UNAM) en el año 2018, por presentar dolor (magnitud 10/10 en la escala de valor análoga) y úlceras intraorales sin cicatrizar, con antecedentes de hipertensión y osteoporosis, tratada con ácido alendrónico, intraoral con dosis de 70 miligramos, una vez a la semana por 3 ańos.

Tabla 1. Cronología de eventos

\begin{tabular}{ll}
\hline \multicolumn{1}{c}{ AÑO } & \multicolumn{1}{c}{ Tratamiento/ Resultados } \\
\hline 2015 & Inicio de padecimiento actual \\
2016 & Biopsia incisional: sin datos malignidad \\
2017 / Enero & Biopsia incisional: sin datos malignidad \\
2017 / Febrero & Biopsia incisional: absceso \\
2017 & Cámara hiperbárica \\
2018 & $\begin{array}{l}\text { Seis extracciones dentales (42, 41, 31, 32, 33 y 34) } \\
\text { Biopsia incisional: osteonecrosis por bifosfonatos } \\
\text { vs. tumor mandibular }\end{array}$ \\
\hline
\end{tabular}

En la exploración clínica se observó la presencia de aumento de volumen en el tercio inferior de la cara. Cavidad oral con mucosas de inadecuada hidratación, con áreas hiperplásicas circundantes a la exposición ósea necrótica, en sector del cuadrante inferior izquierdo (Figura 1). En el cuello se palparon adenopatías en los niveles IB, IIA Y

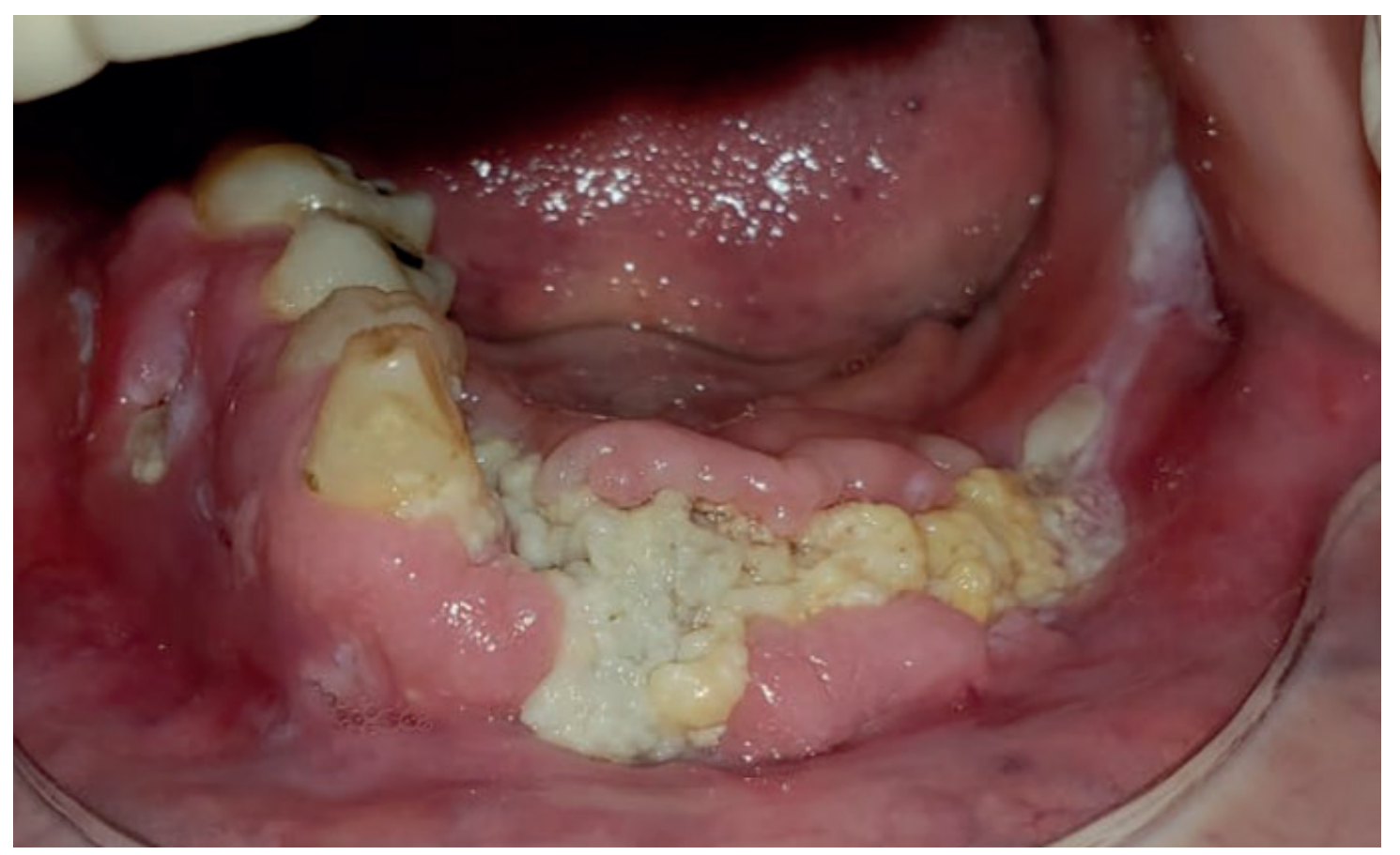

Figura 1. Fotografía intraoral: ausencia de dientes, zona inferior anterior-izquierda con presencia de hueso necrótico expuesto 
IIB según la clasificación de ganglios linfáticos cervicales del American Joint Committee on Cancer, asintomáticos, indurados, de aproximadamente $1 \mathrm{~cm}$. En la ortopantomografía se notó una imagen radiolúcida mal definida (Figura 2). Se decidió realizar biopsia incisional en la cual el resultado fue compatible con carcinoma oral de células escamosas bien diferenciado (Figura $3 \mathrm{~A}, \mathrm{~B}$ y C). A la exploración clínica a los 7 días del postoperatorio se identificó zona tratada de coloración compatible con los tejidos circundantes y presencia de áreas de fibrina. A los 14 días se observó el lecho quirúrgico con coloración no compatible con los tejidos blandos adyacentes y abundante hueso necrótico (Figura 4).

Se remitió la paciente al Instituto Nacional de Cancerología para valoración y tratamiento por COCE, un año y tres meses, posterior a la interconsulta se contacta a la hija de la paciente, refiriendo que se negó a recibir tratamiento (Figura 5).

\section{Discusión}

El COCE es una neoplasia maligna de la cabeza y el cuello, es el sexto cáncer más común en todo el mundo. Está precedido por lesiones asintomáticas tempranas que, si se identifican en etapas tempranas y se eliminan, evitarían la transformación maligna o evitarían retrasar el diagnóstico a etapas avanzadas ${ }^{11}$. La epidemiología del cáncer de cavidad oral refleja fuertemente la exposición a diversos agentes ambientales. El factor de riesgo citado con mayor frecuencia para el cáncer de cavidad oral es el tabaco, que incluye fumar cigarrillos, cigarros o pipas o usar tabaco sin humo.

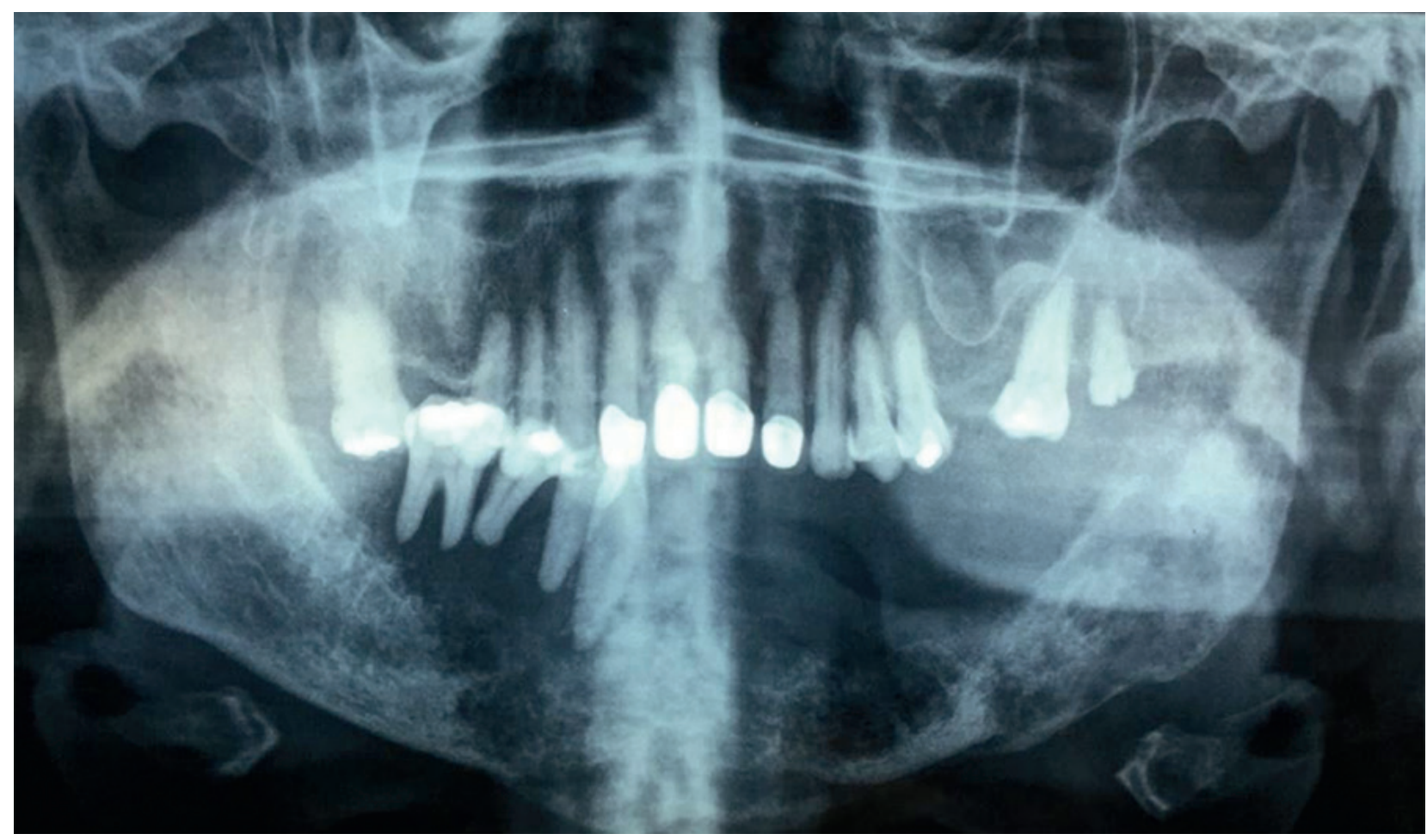

Figura 2. Ortopantomografía, se observan imágenes radiolúcidas de bordes mal definidos, difusos, que involucran cuerpo y rama mandibular
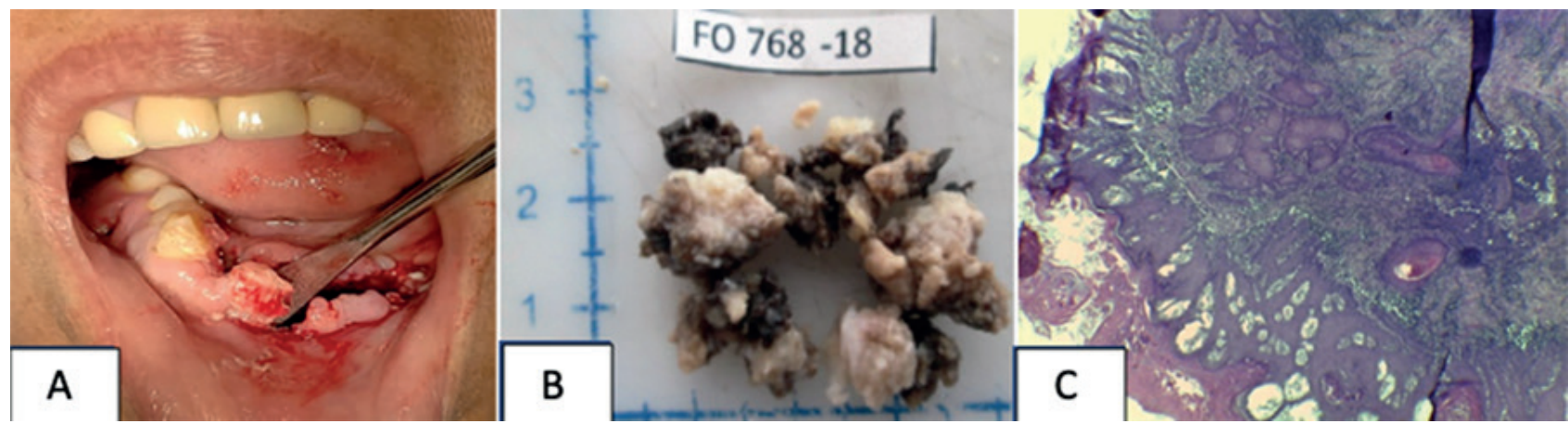

Figura 3. A. Biopsia incisional. B. Examen macroscópico. C. Corte histopatológico se observa una neoplasia maligna de estirpe epitelial con proliferación de células neoplásicas 


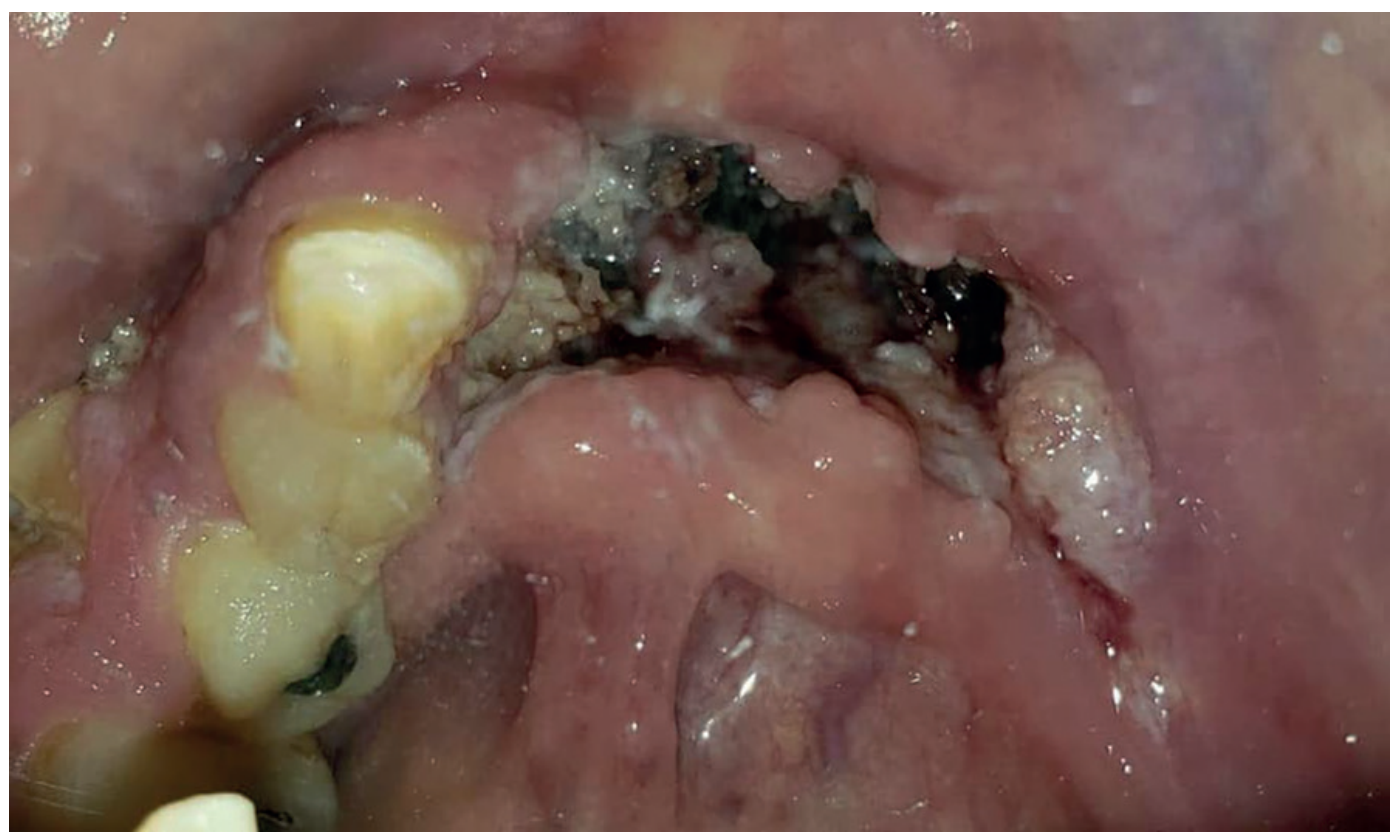

Figura 4. Dos semanas postoperatorio se puede apreciar mayor cantidad de tejido desvitalizado

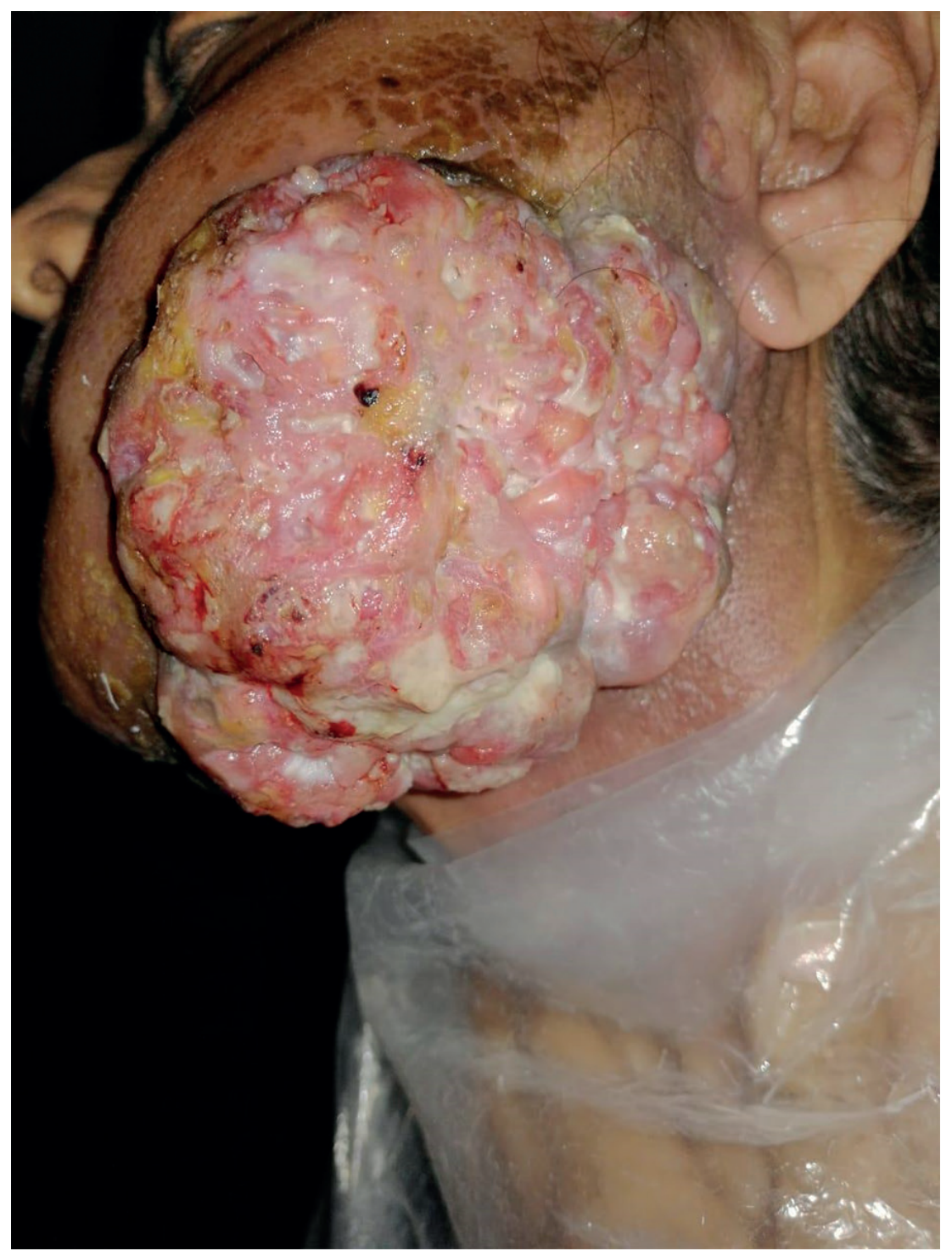

Figura 5. Un año y 3 meses post - operatorio, lesión exofítica, ulceraciones, nódulos superficiales con presencia de necrosis 
El consumo concomitante de alcohol actúa sinérgicamente y aumenta en gran medida el riesgo de desarrollar una neoplasia maligna oral, como en el caso previamente citado la paciente consumió por muchos años de manera continua estas sustancias aumentando su riesgo de padecer cáncer. Las nueces de betel, comúnmente masticadas en el sudeste asiático y la India, también tienen un alto potencial tumorigénico en la cavidad oral. $\mathrm{Nu}$ merosos estudios han demostrado que el uso excesivo de tabaco, junto con el alcohol, son los principales factores de riesgo para el desarrollo de COCE en cabeza y el cuello. El tabaco y el alcohol aumentan el riesgo de una manera dosis-respuesta y sinérgica, los grandes bebedores y fumadores tienen 38 veces más riesgo de desarrollar COCE que la población general ${ }^{12}$.

Otros factores de riesgo reportados para el cáncer de cavidad oral incluyen: irritación crónica por mala higiene oral o sífilis. Las cepas de VPH 16 son responsables de al menos el $90 \%$ de carcinomas orales, la cual es una infección de transmisión sexual, que contribuye a un aumento constante en un subconjunto de neoplasias originadas en la cavidad oral ${ }^{13}$. La irritación mecánica crónica es el resultado de lesiones repetidas por la acción mecánica de un agente de lesión intraoral, dientes defectuosos mal posicionados o con superficies afiladas, dentaduras mal ajustadas y / o hábitos parafuncionales como mordida de la mucosa oral o succión, interposición de la lengua actuando individualmente o en conjunto, podrían ser responsables de cualquier irritación mecánica ${ }^{14}$.

El labio es el sitio más frecuente de cáncer oral en varias áreas geográficas en todo el mundo seguido de la lengua y el piso de boca, y representa el $25 \%-60 \%$ de todos los casos de cáncer de cavidad oral.

En México hay pocos informes de descripción nacional de COCE en cuanto a incidencia o predominio, el cual requiere de un análisis que consista en género, edad de predominio entre la cuarta y septima década de vida y zona predominante de la patología ${ }^{8}$.

Existen datos de lesiones malignas en cavidad oral en México como los reportados por el Dr. Carrillo Rivera ${ }^{16}$ quien notificó 1389 casos de neoplasias en cavidad oral y orofaringe en el hospital general de México. En otro estudio realizado por Hernández Guerrero ${ }^{16}$; con un total de 531 pacientes diagnosticados con COCE entre 1990 y 2000 ; presentó que los pacientes con más de 40 años tuvieron más recurrencia en esta patología $(88,3 \%)$. El sexo predominante fue el masculino con un $58,4 \%$ y la zona de mayor predominio fue la lengua con un 44,7\%. Del 2000 al 2008 en el Hospital General de México (HGM) fueron recibidos 654270 pacientes de los cuales 4925 fueron diagnosticados con COCE ${ }^{12}$. Un estudio realizado en Taiwán demostró que las tasas de supervivencia global a cinco años de COCE para las etapas I, II, III y IV fueron 78,98\%, 69,38\%, 54,62\% y $36,17 \%$, respectivamente ${ }^{1}$

Un método de diagnóstico es la termografía es una técnica para medir la temperatura de una superficie, es capaz de detectar, en tiempo real, cambios en los patrones de temperatura relacionados con procesos inflamatorios, así como condiciones endocrinas y oncológicas. Los tumores surgen del desarrollo incontrolado de células malignas en el cuerpo, lo que resulta en un aumento de la perfusión sanguínea en la región afectada, lo que provoca un aumento de la temperatura local. Este aumento de temperatura tiene el potencial de ser detectado en imágenes termográficas. Además, los cambios en la temperatura del tumor a lo largo del tiempo pueden respaldar la definición de estrategias de tratamiento ${ }^{15}$.

Es de suma importancia que los profesionales del área de la salud y en especial los cirujanos maxilofaciales se familiaricen con el diagnóstico precoz del COCE y todas aquellas lesiones potencialmente malignas. Es fundamental realizar un correcto tamizaje para cáncer oral y frente a sospecha de patologías neoplásicas saber que una biopsia es necesaria y que sólo así se podrá dar un tratamiento precoz, ya que este depende en definitiva del primer profesional que ve la lesión.

\section{Referencias bibliográficas}

1. Ribeiro IP, Barroso L, Marques F, Barbosa J, Marques I. Early detection and personalized treatment in oral cancer: the impact of omics approaches. Mol Cytogenet. [Internet] 2016;9(85):7 páginas.

2. Vazquez J. Estudio comparativo entre la disección cervical supra homohioidea y la biopsia de los ganglios centinela en estadios T1- T2 del carcinoma oral de células escamosas [tesis doctoral]. [Asturias]: Universidad de Oviedo;2013. 222 p.

3. Pavani N, Srinivas P, Kothia N, Chandu V. Recent Advances in the Early Diagnosis of Oral Cancer: A Systematic Review. Int J Med Rev [Internet]. 2017;4(4):119125.

4. Baykul T, Yilmaz HH, Aydin Ü, Aksay M, Yildrim D. Early diagnosis of oral cancer. J. Int Med Res. 2010;38:737-749.

5. Bailey JS, Blanchett RH. Management of oral squamous cell carcinoma treated with inadequate excisional biopsy. Am Assoc Oral Maxill Surg. 2001;59(9):1007-10.

6. Zeeba SJ, Safia R, Apoorva H, Sujuta J. Toward early diagnosis of oral cancer: Diagnostic utility of ortomorphological features, a pilot study. Natl J Maxillofac Surg. 2019;10(1):20-6.

7. Carrillo J, Simón N, Gil G, Rodríguez R. Cáncer oral en México. Revisión bibliográfica y presentación de caso. Rev. AMCBM. 2011;7(3):104-108.

8. Cheng CH, Chia N, Chía HC, Jing SH, Sen- tien T, Jung- Der W. Cost-effectiveness analysis of the oral cancer screaming program in Taiwan, Oral Oncol. 2019;89:59-65.

9. Debolina C, Chandrase K, Amitava M. Advances in oral cancer detection. Adv Clin Chem. 2019;91:181-200.

10. Corbin J, Michael JM, Choe J, Kahmke R, Mowery I, Saloma J. Cancers of the oral cavity: Diagnosis and Treatment. Encyclopedia of Cancer; 3rd edition; 2018.

11. Sánchez S, Juárez T, Espinel B, Mould J, Gómez H, De la Fuente J, et.al. Hospital disonarges for oral cancer in 
Mexican Institute of Social Securuty 1991-2000. Rev Med Ins México Seguro Soc. 2008;46:101-108.

12. Mermoda M, Adamb A, Clairc C, Faouzib M, Simona C, Daeppenb JB, et al. Squamous cell carcinoma of the head and neck - screening in patients who misuse alcohol and tobacco in Switzerland: a prospective pilot study. Br J Oral Maxillofac Surg. 2019;57(10):10531057.

13. Rebolledo M, Arango H, Rebolledo R, Alonso I. Rol del virus del papiloma humano en el desarrollo de carcinoma oral: Una revisión. Av Odontoestomatol. 2016;32(3):135-144.
14. Piemote E, Lazos J, Belardinelli P, Secchi P, Brunotto M, Lanfranchi $\mathrm{H}$. Oral cancer associated with chronic mechanical irritation of the oral mucosa. Med Oral Pathology Oral Cir Bucal. 2018;23(2):51-60.

15. Abreu A, Lima T, Meireles A, Souza B, Gonzalez L. Evaluation of surface temperature of tongue for screening of patients with suspected oral cancer. For Science. 2019;7(2):e00639.

16. Hernández J, Jacinto A, Jiménez F, Macario M, Hernández F, Alcántara A. Prevalece trends of oral squamus cell carcinoma México City’s General Hospital experience. Med Oral Patol Oral Cir Bucal. 2013;18(2):e306-311. 